\title{
Cystic Hygroma of the Neck: Ultrasound Findings
}

\author{
Marco Di Serafino ${ }^{*}$, Carmela Mercogliano ${ }^{2}$, Rosa Severino ${ }^{3}$, Francesco Lisanti ${ }^{4}$, \\ Francesco Esposito ${ }^{5}$, Rosario Rocca1, Rosaria Abate ${ }^{2}$, Tonino Cavallo ${ }^{1}$ \\ ${ }^{1}$ Radiology Department, San Carlo Hospital, Potenza, Italy \\ ${ }^{2}$ Women and Child Department, San Carlo Hospital, Potenza, Italy \\ ${ }^{3}$ Radiology Department, Federico II University Hospital, Naples, Italy \\ ${ }^{4}$ Emergency-Urgency Department, San Carlo Hospital, Potenza, Italy \\ ${ }^{5}$ Radiology Department, Santobono-Pausilipon Children Hospital, Naples, Italy \\ Email: "marco.diserafino@ospedalesancarlo.it
}

Received 18 May 2016; accepted 6 June 2016; published 9 June 2016

Copyright (C) 2016 by authors and Scientific Research Publishing Inc.

This work is licensed under the Creative Commons Attribution International License (CC BY).

http://creativecommons.org/licenses/by/4.0/

(c) (i) Open Access

\begin{abstract}
This is a report of a case of cystic hygroma of the neck in a female child. Cystic hygroma is a rare congenital malformation of the lymphatic system, most frequently detected in the head and neck region. Ultrasound is considered as being the first level study to investigate a suspected mass suggestive of cystic hygroma. The Authors describe the main diagnostic ultrasound features for this type of lymphatic lesion.
\end{abstract}

\section{Keywords}

Cystic Hygroma, Ultrasound, Magnetic Resonance Imaging

\section{Introduction}

Lymphangiomas are rare benign malformation of the lymphatic system usually affected children under 2 years of age that can arise in virtually any part of the body provided by lymphatic vessels but most often localized in the head and neck region. The aim of this case report is to present the clinical and imaging findings of an infant with cervical mixed cystic lymphangioma (also known as cystic hygroma), focusing on the rule of the ultrasound (US) and its diagnostic appearance.

"Corresponding author.

How to cite this paper: Di Serafino, M., Mercogliano, C., Severino, R., Lisanti, F., Esposito, F., Rocca, R., Abate, R. and Cavallo, T. (2016) Cystic Hygroma of the Neck: Ultrasound Findings. Open Journal of Radiology, 6, 121-124.

http://dx.doi.org/10.4236/ojrad.2016.62018 


\section{Description of the Case}

A 5-month-old female child presented with complaints of swelling in the left side of the neck previous 2 months and referred to gradually increased in size after common viral upper airways infection. There were no other complaints and not other relevant prenatal or postnatal medical history. Physical examination determined a mass lesion in the left cervical region reaching approximately $5 \mathrm{~cm}$ in size, slightly mobile and pressurized, nontender, non-pulsatile, with no sign of inflammation, leading to posture impairment of the neck. Routine laboratory results were normal. Ultrasound examination of the mass was first performed and showed an irregular multi-loculated compressible cystic lesion of $5 \times 3 \mathrm{~cm}$ in size with thin septa internal debris (Figure 1), not vascularized on colour-Doppler evaluation (Figure 2), located in the left anterior cervical region and extending to the posterior triangle of neck, suggestive of cystic hygroma. No extension or involvement of parotid gland was seen. Small, enlarged nodes were also seen in the upper part of the neck with reactive appearance on US. Magnetic Resonance Imaging (MRI) with contrast agent administration was also made to evaluate the extent of the lesion and details of the internal cystic structure to rule out malignancy. From MRI examination the lesion was determined to be located on the left half of the neck in the supra- and infra-hyoid region, extended in the posterior cervical triangle and surrounding the internal jugular vein, with typical, not contrasted, multi-cystic pattern characterize by low signal intensity on T1-weighted images and high hyperintensity on T2-weighted images (Figure 3). Surgical excision was performed without any vital structure damage and the specimen received was soft, large cystic sac with histologic pattern distinctive of cystic hygroma. At 1-month and 3-month later postoperative follow-up, the patient was asymptomatic and ultrasound was normal without recurrence of lesion.

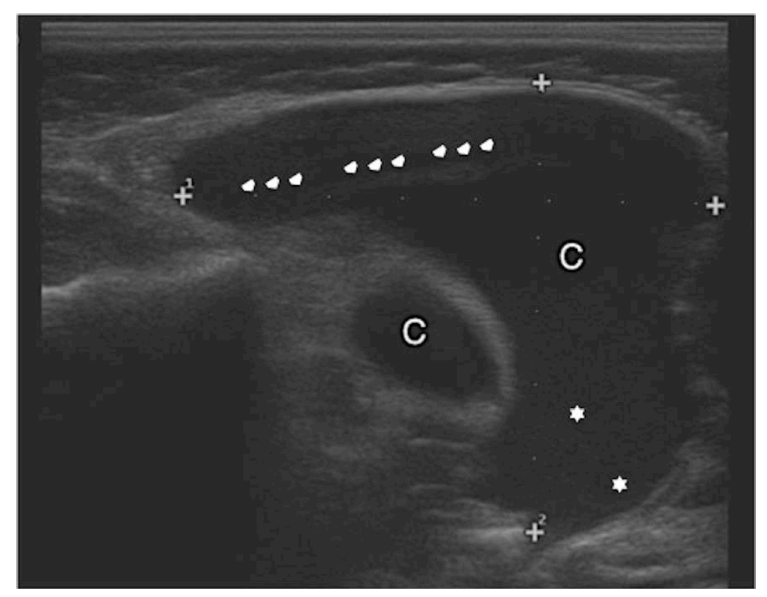

Figure 1. High linear frequency (10 - $15 \mathrm{MHz})$ ultrasound probe on longitudinal medium-caudal left side scan of the neck shows a hypoechoic multicystic (C), trans spatial lesions, with thin septae (arrowheads) and internal debris (stars).

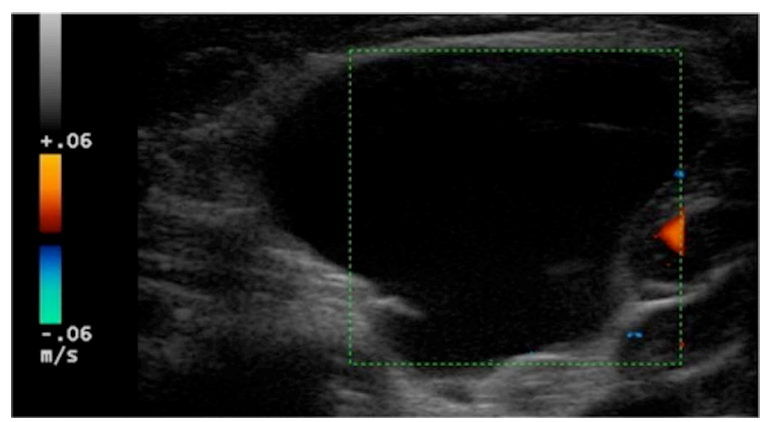

Figure 2. High linear frequency (10 - $15 \mathrm{MHz}$ ) ultrasound probe on transverse cranial left side scan of the neck with colour - Doppler module shows a hypoechoic cystic lesion (C) with an absence of identifiable flow signal. 


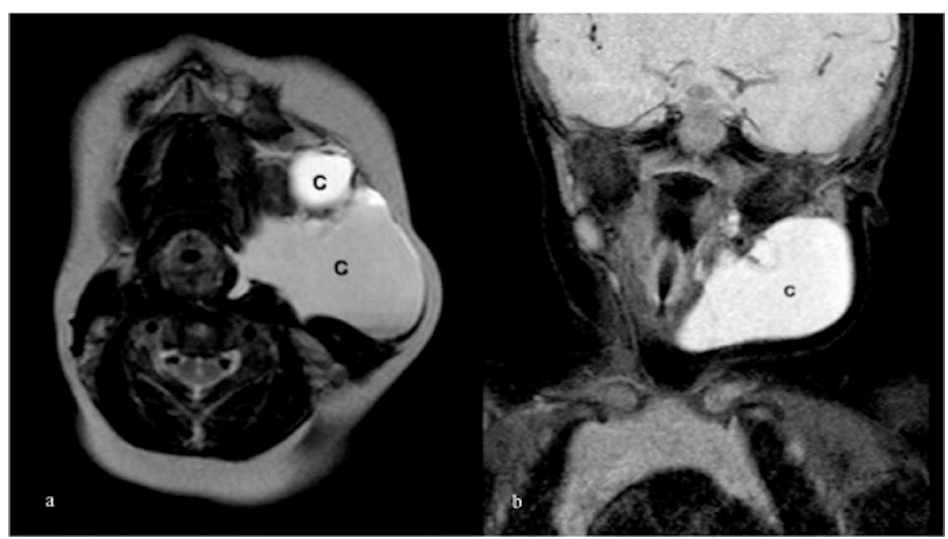

Figure 3. Axial TSE (a) and coronal STIR (b) T2-weighted MRI images show a hyperintense multiloculated cystic mass (C) located on the left half of the neck in the infra-suprahyoid region, extending into the retropharyngeal cavity to the posterior cervical triangle with not significant compression effect.

\section{Discussion}

Lymphangiomas are unusual vascular lesions of uncertain origin and represent a spectrum of lesions usually classified into three pathologic subtypes by size of dilated lymphatic channels within: microcystic, macrocystic and mixed cystic (or cystic hygromas) [1]-[3]. They are generally considered vascular malformations resulting from sequestrations of lymphatic tissue that fail to communicate normally with the lymphatic system and occur at the original sites of the primordial lymph sacs in fetuses, neonates, or young children [1]. The incidence of lymphangiomas has been reported to range from 1.2 to 2.8 per 1000 new-borns [2]. The cystic form consists of macrocystic dilatations and when localized in the head-neck region (75\%), it is referred to as a cystic hygroma and is caused by a developmental lag in jugular lymph-sac drainage into the jugular vein [4]. Usually cystic hygromas arise in areas such as neck and axilla, where loose connective tissue allows expansion of the endotheliallined channels, instead, cavernous lymphangiomas, develop in the mouth, lips, cheek, tongue or other areas where dense connective tissue and muscles prevent expansion [5] [6]. Cystic hygromas, account for approximately $90 \%$ of the lymphangiomas in the head and neck region and more frequently located on the left side, as our case. Other common sites, outside the head and neck, include the axilla, shoulder, chest wall, mediastinum, abdominal wall, and thigh [2]. Most cystic hygromas are diagnosed in the period immediately after birth, with large lesions being usually noted on prenatal ultrasonography. Later presentations generally follow a viral infection, which can induce significant expansion of these lesions, as our case [7].

The most prominent sign or symptom of all lymphangiomas is the presence of a mass with associated symptoms of respiratory obstruction and problems with feeding [2].

Clinically, the skin is usually normal but may have bluish hue, puckering or tiny vescicles. These lesions can be diffuse or focal tend to invade multiple anatomic facial planes and will rapidly enlarge if complicated by haemorrhage or infections. Hypertrophy and intraosseous invasions of adjacent bone, usually the mandible can occur [8]. Although most children with lymphatic malformations have a normal karyotype, several syndromes are associated (e.g. Turner, Down, Noonan, Roberts), as well as trisomy 13 and 18 [8]. The lymphangiomas are slow-growing with not malignant potential and rarely resolve spontaneously [4]. Surgical resection is the treatment of choice but can be difficult en bloc resection if the lesion invades neural, vascular or muscular structures. Total excision is important for the prognosis, because cyst remnants usually cause tumour recurrence; in addiction sclerosing therapy should be considered as nonsurgical treatment modalities of choice for cystic hygroma specially in the recurrences [2].

Imaging has a main role in the detection and characterization of cystic content either with US and MRI or Computed Tomography (CT) too [9].

The case presented here had a left neck mass lesion after birth and a diagnosis of cystic hygroma was first made with ultrasound. US is considered as being the first level study to investigate a suspected mass suggestive of cystic hygroma because of its non-invasiveness, low cost, and non-use of ionizing radiation [9]. On ultrasound, 
cystic hygromas appearance as multi-loculated cystic compressible mass with thin walls and intervening septa, not vascularized on colour-Doppler, as the case presented. Fluid-fluid levels or echogenic debris can result from superimposed haemorrhage or infection, in this eventuality, the US appearance can be change into not compressible, hypoechoic, heterogeneous mass with thick and vascularized walls and septa and with mass effect that can make the differential diagnosis with vascularized lesion more difficult [10]. US is also the ideal modality to guide injections of sclerosing agent, reducing procedure time and increased safety of procedure, and it is the choice for follow-up after treatment [10]. On US a trans-spatial multicystic neck mass with septation debris and fluid levels is most likely cystic hygroma differentiating from other benign cystic mass of the neck such as $2^{\text {nd }}$ brachial cleft cyst, thymic cyst and tyroglossal duct cyst or abscess [10]. While US scans diagnose lymphangiomas, MRI or CT are necessary to map their entire extent [9] [10]. In particular MRI with contrast agent provides an optimum tissue characterization and anatomy of the lesion with a great benefit before surgical interventions [9] [10].

\section{Conclusion}

In conclusion, as a result of the aforementioned characteristics, cystic hygroma is rather rare vascular lesions and it should be considered in the differential diagnosis of head and neck cystic masses. Ultrasound is considered as being the first level study to investigate a suspected mass suggestive of cystic hygroma. As mentioned in previous studies [9] an appropriate diagnostic imaging protocol in patients with cystic hygromas should initially include an US study with a successive MRI scan with contrast agent administration to further investigate lesion features and to map the extent of cystic hygroma before surgical interventions.

\section{Acknowledgements}

The Authors declare that no conflict of interest exists with the results and conclusions presented in this paper. Publication ethics have been observed.

\section{Informed Consent}

All procedures followed were in accordance with the ethical standards of the responsible committee on human experimentation (institutional and national) and with the Helsinki Declaration of 1975, and its late amendments. Additional informed consented was obtained from all patients for which identifying information is not included in this article.

\section{References}

[1] Licci, S., Puma, F., Sbaraglia, M. and Ascani, S. (2014) Primary Intrathymic Lymphangioma. American Journal of Clinical Pathology, 142, 683-688. http://dx.doi.org/10.1309/AJCPGDRPIMYSSX7U

[2] Grasso, D.L., Pelizzo, G., Zocconi, E. and Schleef, J. (2008) Lymphangiomas of the Head and Neck in Children. Acta Otorhinolaryngologica Italica, 28, 17-20.

[3] ISSVA Classification for Vascular Anomalies (2015) International Society for the Study of Vascular Anomalies (Approved at the 20th ISSVA Workshop, Melbourne, April 2014).

[4] Bengtsson, B. (2013) Neonatal Lymphatic (Chylous) Disorders. NeoReviews, 14, e600-e612. http://dx.doi.org/10.1542/neo.14-12-e600

[5] Bill Jr., A.H. and Sumner, D.S. (1965) A Unified Concept of Lymphangioma and Cystic Hygroma. Surgery, Gynecology \& Obstetrics, 120, 79-86.

[6] Joshi, P.S., Hongal, B. and Sanadi, A. (2015) Cystic Lymphangioma: A Differential Diagnosis. Journal of Oral and Maxillofacial Pathology, 19, 393-395. http://dx.doi.org/10.4103/0973-029X.174622

[7] Geddes, G., Butterly, M.M., Patel, S.M. and Marra, S. (2013) Pediatric Neck Masses. Pediatrics in Review, 34, 115124. http://dx.doi.org/10.1542/pir.34-3-115

[8] Rumack, C.M., et al. (2011) The Pediatric Head and Neck. In: Diagnostic Ultrasound, 4th Edition, Elsevier, Philadelphia, 1718-1719.

[9] Romeo, V., Maurea, S., Mainenti, P.P., Camera, L., Aprea, G., Cozzolino, I. and Salvatore, M. (2015) Correlative Imaging of Cystic Lymphangiomas: Ultrasound, CT and MRI Comparison. Acta Radiologica Open, 4, 1-4. http://dx.doi.org/10.1177/2047981614564911

[10] Ahuja, A.T., et al. (2007) Lymphangioma. In: Diagnostic Imaging Ultrasound, Amirsys, Salt Lake City, 92-97. 